\title{
Strategies for Measurements of Pseudocontact Shifts in Protein NMR Spectroscopy
}

\author{
Michael John ${ }^{[b]}$ and Gottfried Otting ${ }^{*[a]}$
}

Paramagnetic metal ions bound to proteins generate a dipolar field that can be accurately probed by pseudocontact shifts (PCS) displayed by the protein's nuclear spins. PCS are highly useful for determining the coordinates of individual spins in the molecule and for rapid structure determinations of entire protein-protein and protein-ligand complexes. However, PCS measurements re- quire reliable resonance assignments for the molecule in its paramagnetic state and in a diamagnetic reference state. This article discusses different approaches for pairwise resonance assign ments, with emphasis on a strategy which exploits chemical exchange between the two states.

\section{Introduction}

Paramagnetic metal ions provide NMR spectroscopists with unique tools for structural studies of metalloproteins. Since the magnetic moment of a single unpaired electron is 658 times that of a proton, the dipole-dipole interaction between a paramagnetic spin and a nuclear spin (hyperfine interaction) is much stronger and measurable over much larger distances than the dipolar interaction between two nuclear spins. Hyperfine interactions thus cause pronounced effects in the NMR spectrum, including resonance shifts, enhanced longitudinal and transverse relaxation, differential relaxation of individual multiplet components, and modulation of apparent spin-spin coupling constants. ${ }^{[1]}$ All these parameters can be translated into restraints for structure determinations of proteins and their complexes. ${ }^{[2-9]}$

\section{Pseudocontact Shifts}

Resonance shifts in paramagnetic molecules have mainly two sources: Fermi-contact shifts are significant only for nuclear spins within a few chemical bonds of the metal ion, whereas pseudocontact shifts (PCS) are purely dipolar and therefore long-range interactions. ${ }^{[10]}$ PCS can be understood as the consequence of an average dipolar field generated by the paramagnetic center and felt by the nuclear spin at its particular location $\mathbf{r}$ in the molecule [Figure $1 \mathrm{a}$ and Eq. (1)]: ${ }^{[1]}$

$\Delta \delta^{\mathrm{PC}}=\delta^{\mathrm{PARA}}-\delta^{\mathrm{DIA}}=\frac{1}{12 \pi r^{5}} \operatorname{Tr}\left\{3 \mathbf{r} \otimes(\mathbf{r} \boldsymbol{\chi})-r^{2} \boldsymbol{\chi}\right\}$

where $\delta^{\text {PARA }}$ and $\delta^{\text {DIA }}$ are the nuclear chemical shifts in the presence and absence of a paramagnetic metal ion, respectively, and $\chi$ is the magnetic susceptibility tensor which is centered on the metal ion and fixed in the molecular frame. Note that $\Delta \delta^{\mathrm{PC}} 1$ ) becomes zero for metal ions with isotropic $\chi$ (i.e. for $\mathrm{Mn}^{2+}$ or $\mathrm{Gd}^{3+}$ ) and 2) is independent of the static magnetic field or nuclear spin type.
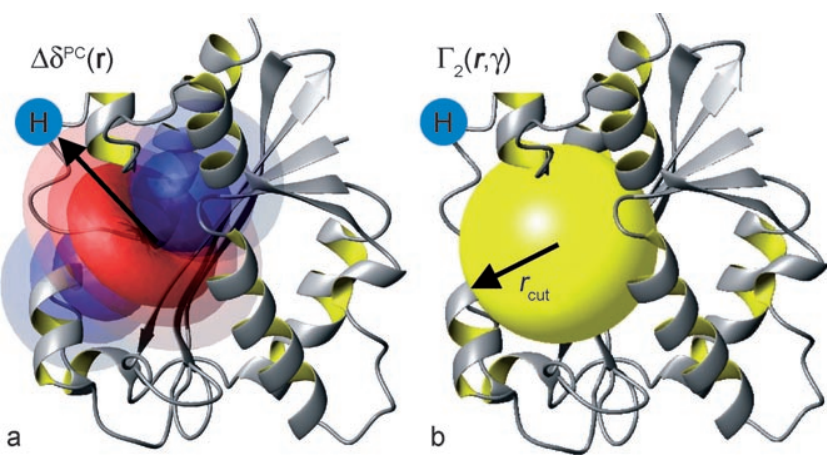

Figure 1. PCS and PRE in a paramagnetic protein. a) $\mathrm{PCS}\left(\Delta \delta^{\mathrm{PC}}\right)$ are induced by the dipolar field at the position $\mathbf{r}$ of the nuclear spin (here denoted " $\mathrm{H}$ ") relative to the paramagnetic center. Blue and red regions show where the dipolar field induces positive and negative PCS, respectively. b) PRE $\left(\Gamma_{2}(r, \gamma)\right)$ depend on the distance $r$ from the paramagnetic center and on the nuclear magnetogyric ratio $\gamma$. Nuclear spins with $r<r_{\text {cut }}$ (i.e. within the yellow sphere) are not observable in a given NMR experiment due to strong PRE.

Once the magnitude of $\chi$ and its orientation with respect to the molecular frame is known, PCS can be back-calculated for every nuclear spin from its coordinates. This opens exciting possibilities for structural studies in which PCS are used along with other constraints. ${ }^{[11]}$ As PCS are less sensitive to local dynamics than most other NMR parameters they are particularly helpful for regions in the protein that are poorly defined by conventional NMR data. ${ }^{[2]}$ Even if the molecular structure is already known, PCS can highlight small atomic displacements ${ }^{[12]}$ and dynamic behavior. ${ }^{[13,14]}$ Furthermore, they can be used to

\footnotetext{
[a] Prof. G. Otting

Research School of Chemistry, Australian National University Canberra ACT 0200 (Australia)

Fax: (+61) 02-6125-0750

E-mail:go@rsc.anu.edu.au

[b] Dr. M. John

Institut für Anorganische Chemie

Georg August Universität, Tammannstrasse 4

37073 Göttingen (Germany)
} 


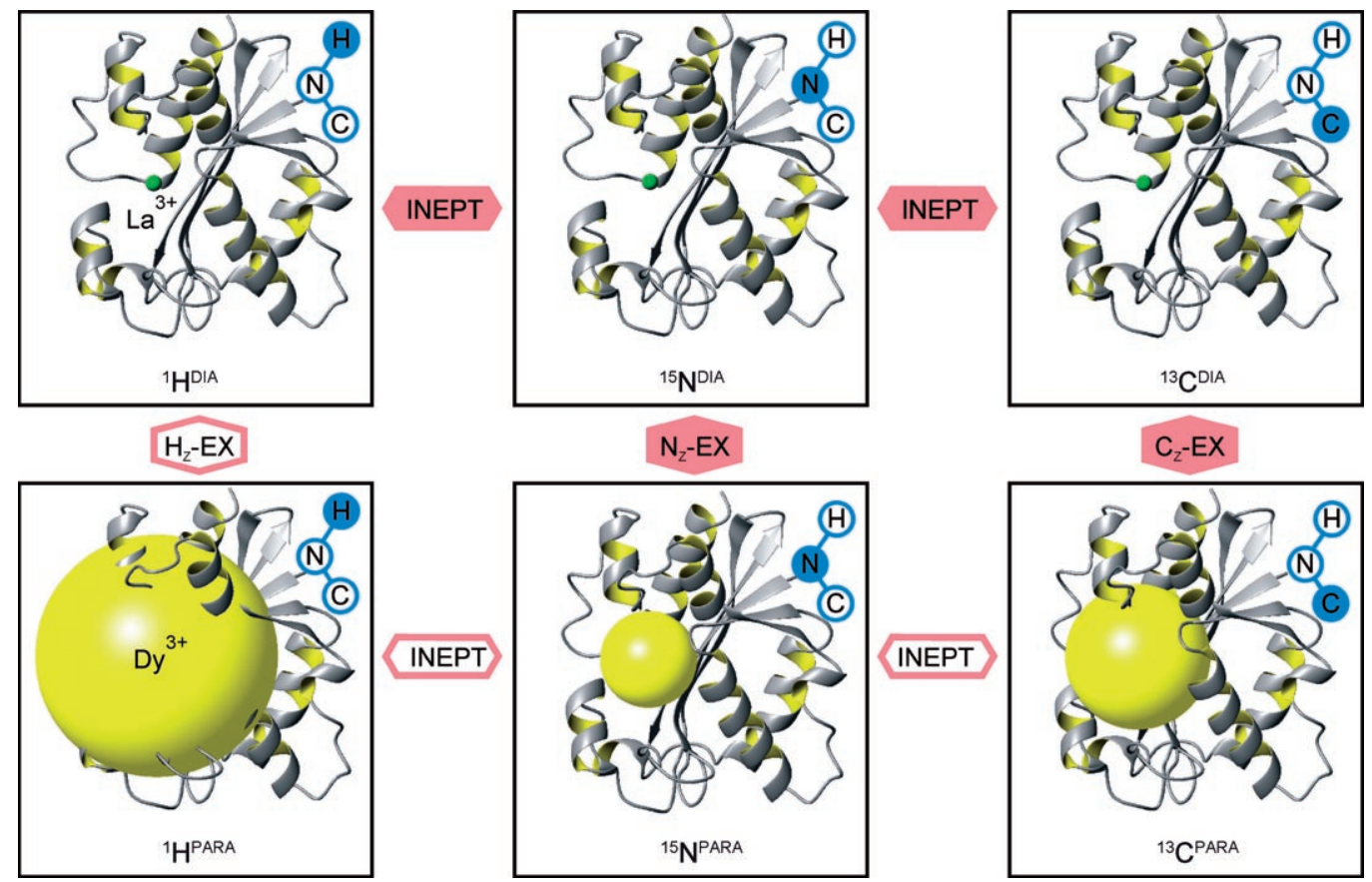

Figure 2. Magnetization transfer in diamagnetic and paramagnetic proteins. Conventional INEPT-based methods can be used to connect ${ }^{1} \mathrm{H}$, ${ }^{15} \mathrm{~N}$ and ${ }^{13} \mathrm{C}$ spins and achieve sequential resonance assignments in the diamagnetic state (illustrated here with a $\mathrm{La}^{3+}$ ion) and for spins far from the metal ion in the paramagnetic state $\left(\right.$ e.g. $\left.\mathrm{Dy}^{3+}\right)$. Connections between the states can be established directly by chemical exchange or inderectly by one of the methods described in the text. The yellow spheres mark typical $r_{\text {cut }}$ boundaries within which NMR signals are completely lost due to excessive PRE (15 $\AA$ for ${ }^{1} \mathrm{H}, 6 \AA$ for ${ }^{15} \mathrm{~N}$, and $9 \AA$ for $\left.{ }^{13} \mathrm{C}\right)$.

aid in resonance assignments ${ }^{[15,16]}$ or the structure determination of larger molecular assemblies (Figure 2)..$^{[3,5-7,9]}$

\section{Paramagnetic Relaxation Enhancement}

Enhanced transverse relaxation of nuclear spins is concomitant with a broadening of NMR resonances and therefore a rather undesired effect of the paramagnetism. Paramagnetic relaxation enhancement (PRE) arises from random modulations of the dipolar field due to molecular tumbling or electronic transitions within the paramagnetic center. In macromolecules with electronically fast relaxing metal ions, PRE is predominantly induced by tumbling (Curie relaxation) ${ }^{[17]}$ and approximately given by [Eq. (2)]:

$\Gamma_{2}=R_{2}^{\text {PARA }}-R_{2}^{\text {DIA }}=\frac{1}{5 \pi r^{6}} \gamma^{2} B_{0}^{2} \chi^{2} \tau_{c}$

where $R_{2}^{\text {PARA }}$ and $R_{2}$ DIA denote the transverse relaxation rates in the paramagnetic and diamagnetic states, respectively. Equation (2) shows that, in contrast to PCS, PRE depends on the nuclear magnetogyric ratio $\gamma$, the static magnetic field $B_{0}$, and the molecular rotational correlation time $\tau_{c}$. Most important, however, PRE scales with $r^{-6}$ (as opposed to the $r^{-3}$ dependence of PCS) and therefore adopts very large values for nuclear spins in the vicinity of the metal ion. This means that PCS can be measured only for nuclear spins located beyond a minimal cutoff distance $r_{\text {cut }}$ of the paramagnetic center. For spins with $r<r_{\text {cut }}$ PCS and, hence, the associated structural information remain inaccessible (Figure $1 \mathrm{~b}$ ).

\section{PCS Measurements}

The PCS value of a given nuclear spin can be measured experimentally as the difference of its chemical shift between the states where the protein is bound to the paramagnetic metal ion and a diamagnetic reference. In principle, the metal-free form of the protein can serve as the diamagnetic reference, but, due to the possibility of conformational changes caused by the metal binding, a reference containing a diamagnetic metal ion with similar chemical properties is preferrable. In either case PCS measurements require the resonance assignments in both states or at least a pairwise correlation of the NMR signals in the paramagnetic and diamagnetic states. This can be achieved by one of the following methods.

\section{Conventional Resonance Assignment}

The most obvious method of PCS measurements is a separate assignment of as many resonances as possible in the paramagnetic and diamagnetic NMR spectra, followed by calculation of their chemical shift differences [Eq. (1)]. For diamagnetic proteins, sequential resonance assignment is usually achieved by ${ }^{1} \mathrm{H}$-detected, multidimensional NMR experiments that employ INEPT-type magnetization transfer through chemical bonds between ${ }^{1} \mathrm{H},{ }^{15} \mathrm{~N}$ and ${ }^{13} \mathrm{C}$ spins (Figure 2). ${ }^{[18]}$ The approach does not require prior knowledge of the protein structure and has 
been demonstrated for monomeric proteins with a molecular weight of up to $80 \mathrm{kDa}{ }^{[19]}$ For paramagnetic proteins, PRE interferes with the long delays required for magnetization transfer across small scalar couplings. Therefore, these experiments are usually applicable only to residues far away from the paramagnetic center (large $r$ ) or for very weak paramagnetic ions (small $\chi$; Figure 3). ${ }^{[20]}$

\section{Protonless Detection}

A substantial reduction of $r_{\text {cut }}$ and therefore extension of the number of observable residues can be achieved by a series of protonless NMR experiments that have been proposed recently. ${ }^{[21]}$ Due to the $\gamma^{2}$ dependence of PRE [Eq. (2)], $r_{\text {cut }}$ is, under otherwise identical conditions, theoretically 1.6-fold smaller for
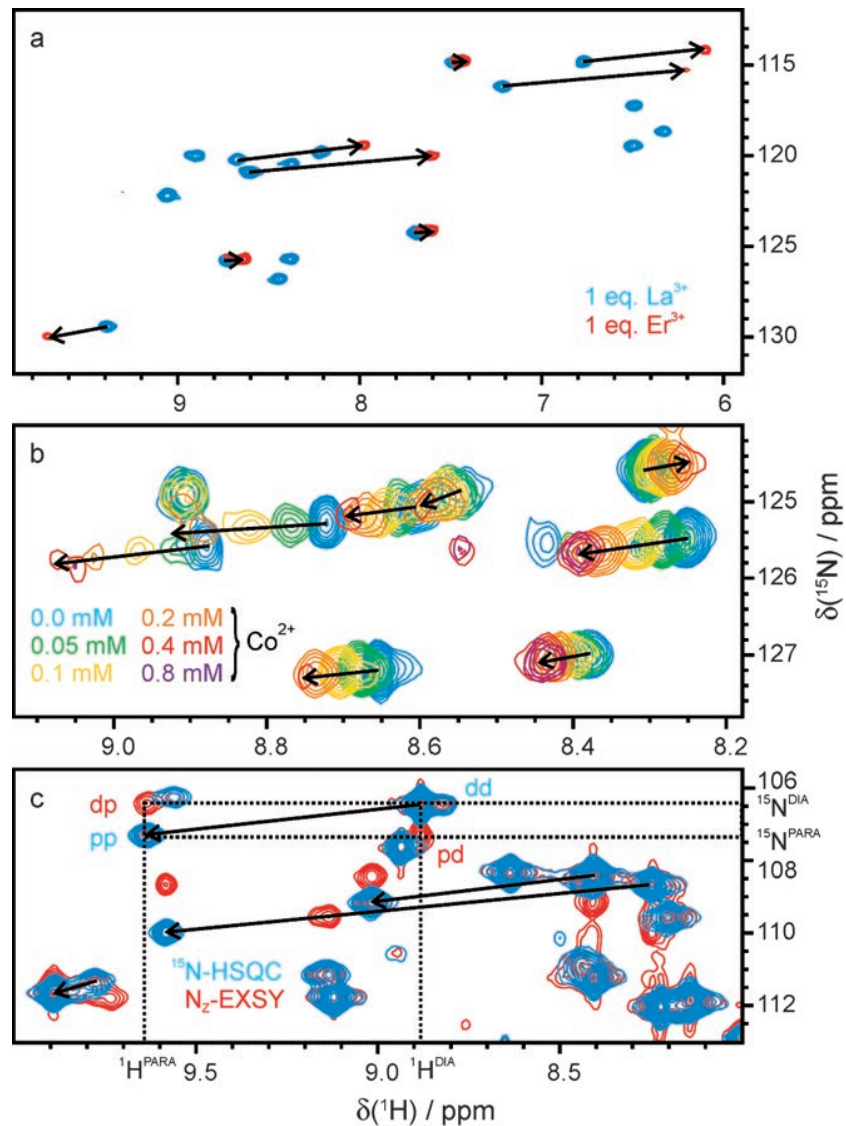

Figure 3. Different approaches for PCS measurements. a) Using the similarity of ${ }^{1} \mathrm{H}$ and ${ }^{15} \mathrm{~N}$ PCS which leads to peak displacements along (almost) parallel lines, as illustrated by ${ }^{15} \mathrm{~N}-\mathrm{HSQC}$ spectra of the $\varepsilon 186 / \theta$ complex in the presence of $\mathrm{La}^{3+}$ (blue peaks) and $\mathrm{Er}^{3+}$ (red peaks). Only Leu residues of $\varepsilon 186$ are ${ }^{15} \mathrm{~N}$ labeled. Missing peaks of the paramagnetic state belong to amide groups with $r<15 \AA$. b) Using the increasing chemical shift changes upon titration with paramagnetic metal ions, as illustrated by ${ }^{15} \mathrm{~N}-\mathrm{HSQC}$ spectra of $\varepsilon 186 / \theta$ with increasing concentrations of $\mathrm{Co}^{2+}$. At $0.4 \mathrm{~mm}$ (red peaks), the active site is essentially saturated with $\mathrm{Co}^{2+}$, so that additional $\mathrm{Co}^{2+}$ greatly broadens the peaks due to unspecific binding. c) Using slow chemical exchange between paramagnetic and diamagnetic metal ions, as illustrated by the overlay of ${ }^{15} \mathrm{~N}-\mathrm{HSQC}$ (blue peaks) and $\mathrm{N}_{\mathrm{Z}}$-EXSY (red peaks) spectra of $\varepsilon 186 / \theta$ in the presence of a 1:1 mixture of $\mathrm{La}^{3+}$ and $\mathrm{Dy}^{3+} \cdot{ }^{15} \mathrm{~N}-\mathrm{HSQC}$ peaks are labeled "dd" and "pp" for the diamagnetic and paramagnetic states, respectively. Exchange peaks are labeled "dp" and "pd" for transitions ${ }^{15} \mathrm{~N}^{\mathrm{DIA}} \rightarrow$ ${ }^{15} \mathrm{~N}^{\text {PARA }}$ and vice versa.
${ }^{13} \mathrm{C}$ than for ${ }^{1} \mathrm{H}$ spins (and 2.2 -fold smaller for ${ }^{15} \mathrm{~N}$ spins, Figure 2). Note that, in practice, $r_{\text {cut }}$ also depends on the particular NMR experiment, since the impact of PRE increases with the time during which the spins are subject to transverse relaxation. For example, an amide proton resonance of $70 \mathrm{~Hz}$ line width may already escape detection in a ${ }^{15} \mathrm{~N}-\mathrm{HSQC}$ experiment, where the magnetization transfer from ${ }^{1} \mathrm{H}$ to ${ }^{15} \mathrm{~N}$ and back requires two INEPT periods of $5.5 \mathrm{~ms}$ each (Figure 2).

\section{Similarity of PCS of Nearby Spins}

Since the vector $\mathbf{r}$ is similar for spins that are separated by only one chemical bond, their PCS are similar, and cross-peaks between those spins are displaced along parallel lines in the spectrum. Figure 3 a shows that this criterion may be sufficient to identify a limited number of pairs of diamagnetic and paramagnetic peaks. In the case of a known protein structure, the associated PCS values can be used to obtain the $\chi$ tensor and to predict the PCS also for nuclei closer to the metal ion where the peak displacement depends more strongly on the orientation of the internuclear vector. ${ }^{[22]}$ This approach can be automated, yielding paramagnetic assignments for uniformly ${ }^{15} \mathrm{~N}$-labeled proteins from simple two-dimensional spectra. ${ }^{[23]}$ For proteins with well-resolved spectra as in Figure $3 \mathrm{a}$, programs are available that assign both the paramagnetic and the diamagnetic NMR spectrum by reference to the $3 D$ structure of the protein. ${ }^{[16,24]}$

\section{Temperature and Field Dependence of PCS}

Connectivities between diamagnetic and paramagnetic NMR resonances can also be established experimentally. Provided $\chi$ scales approximately linearly with the inverse temperature $T^{-1}$ (Curie behavior), the paramagnetic peak positions measured as a function of the temperature can be extrapolated to infinite $T$ to yield the diamagnetic peak position. ${ }^{[25]}$ Similarly, saturation of magnetic moments at large magnetic fields results in a $B_{0}{ }^{-}$ dependence of $P C S^{[26]}$ that may be exploited for resonance assignments. This method may not be generally applicable as it requires the protein to be stable over a large temperature range or the availability of a large range of magnetic fields. The temperature approach can be severely impeded by temperature dependent chemical shifts in the diamagnetic state.

\section{Fast Metal Exchange}

Another method of scaling the effective $\chi$ tensor is applicable to situations where the binding of the paramagnetic metal to the protein is kinetically labile. This is illustrated in Figure $3 \mathrm{~b}$ with ${ }^{15} \mathrm{~N}-\mathrm{HSQC}$ spectra of the $\varepsilon 186 / \theta$ complex of DNA polymerase III ${ }^{[27]}$ that weakly $\left(K_{\mathrm{d}} \sim 0.1 \mathrm{~mm}\right)$ binds $\mathrm{Co}^{2+}$ in the active site. Binding and dissociation of $\mathrm{Co}^{2+}$ ions occurs at a rate $k_{\text {ex }}$ that is much larger than the frequency differences $\Delta \omega(=$ $\gamma B_{0} \delta^{\mathrm{PC}}$ ) between the paramagnetic and diamagnetic state, so that the amide resonances appear at average positions determined by the relative populations of the two states. Therefore, the peaks gradually shift with increasing metal concentration 
from the diamagnetic to the paramagnetic position, and PCS can be measured by extrapolation or from the shift observed when the protein is fully saturated with $\mathrm{Co}^{2+}$. The drawback of this method is that unspecific and specific binding may compete at the metal concentrations required for saturation, causing unwarranted line broadening that interferes with the PCS measurement.

\section{Slow Metal Exchange}

Most metalloproteins bind metal ions rather tightly, so that dissociation is slow on the NMR timescale and discrete resonances are observed for the diamagnetic and paramagnetic states. Slow processes may, however, become detectable by exchange spectroscopy (EXSY), if they are sufficiently rapid to compete with nuclear relaxation during a mixing period. ${ }^{[28]}$ In a suitable exchange regime, EXSY therefore generates exchange peaks that can be used to correlate diamagnetic with paramagnetic resonances. In macromolecules, longitudinal magnetization commonly used during the mixing period fortunately relaxes particularly slowly and is also insensitive to PRE. ${ }^{[29]}$

The well-known ${ }^{1} \mathrm{H}$-EXSY experiment ${ }^{[28]}$ has been used for this purpose, ${ }^{[30]}$ but is not practical for macromolecules where exchange is difficult to distinguish from cross-relaxation (NOE) between nearby protons. Much more suitable in this respect are ${ }^{15} \mathrm{~N}$ and ${ }^{13} \mathrm{C}$ spins whose longitudinal $\left(\mathrm{N}_{\mathrm{z}}, \mathrm{C}_{\mathrm{Z}}\right)$ magnetization is inherently long-lived and which provide additional signal dispersion in the NMR spectra. For example, the $\mathrm{N}_{\mathrm{z}}$-EXSY experiment is a two-dimensional NMR experiment with a ${ }^{15} \mathrm{~N}-\mathrm{HSQC}$ like appearance that has been originally developed to study a slow folding-unfolding equilibrium in a protein fragment. ${ }^{[31]}$

The $\mathrm{N}_{\mathrm{Z}}$-EXSY experiment has recently been applied in our laboratory to the $\varepsilon 186 / \theta$ complex in the presence of a $1: 1 \mathrm{mix}$ ture of diamagnetic (e.g. $\mathrm{La}^{3+}$ ) and paramagnetic (e.g. Dy ${ }^{3+}$ ) lanthanides (Figure $3 \mathrm{c}$ ). ${ }^{[29]}$ In this case, the $\mathrm{N}_{\mathrm{z}}$-EXSY experiment creates exchange cross-peaks from the unidirectional magnetization pathway ${ }^{1} \mathrm{H}^{\text {DIA }} \rightarrow{ }^{15} \mathrm{~N}^{\text {DIA }} \rightarrow{ }^{15} \mathrm{~N}^{\text {PARA }} \rightarrow{ }^{1} \mathrm{H}^{\text {PARA }}$ (" $\mathrm{dp}^{\text {" }}$ path) or vice versa ("pd" path, Figure 2). Either pathway is still affected by ${ }^{1} \mathrm{H}$ PRE during a single INEPT period. In order to further reduce $r_{\text {cut }}$ for PCS measurements, the presence of transverse ${ }^{1} \mathrm{H}$ magnetization in the paramagnetic state has to be completely avoided.

In a recent experiment we have shown that the effective $r_{\text {cut }}$ could be significantly reduced in the $\varepsilon 186 / \theta$ complex by the use of the following out-and-back magnetization transfer pathway: ${ }^{1} \mathrm{H}^{\text {DIA }} \rightarrow{ }^{15} \mathrm{~N}^{\text {DIA }} \rightarrow{ }^{15} \mathrm{~N}^{\text {PARA }} \rightarrow{ }^{15} \mathrm{~N}^{\text {DIA }} \rightarrow{ }^{1} \mathrm{H}^{\text {DIA }} \cdot{ }^{[32]}$ In this experiment, chemical exchange is thus enlisted twice to shuttle ${ }^{15} \mathrm{~N}$ magnetization from the diamagnetic to the paramagnetic state and back. In this way, the ${ }^{15} \mathrm{~N}$ magnetization can be used as a spy to probe the paramagnetic effects close to the metal ion while the sensitivity is maximized by using ${ }^{1} \mathrm{H}$ magnetization during the preparation and detection periods of the pulse sequence. The long lifetime of ${ }^{15} \mathrm{~N}$ magnetization makes this experiment attractive even in situations, where the chemical exchange between metal ions is fairly slow. ${ }^{[29]}$

In the case of the analogous $C_{z}$-EXSY experiment of methyl groups, however, the ${ }^{13} \mathrm{C}^{\text {PARA }}$ spins are observed with better sensitivity in an experiment designed with a unidirectional path ${ }^{13} \mathrm{C}^{\text {PARA }} \rightarrow{ }^{13} \mathrm{C}^{\mathrm{DIA}} \rightarrow{ }^{1} \mathrm{H}^{\mathrm{DIA}}$, as the polarization of methyl groups can be efficiently enhanced by ${ }^{1} \mathrm{H}$ presaturation without invoking an INEPT transfer step. ${ }^{[2]}$

\section{Conclusions}

In conclusion, the choice between different methods for PCS measurements in paramagnetic proteins very much depends on the specific system under investigation, in particular, whether 1) the protein structure is known, 2) the paramagnetic center is made by a metal with favourable magnetic properties as provided by lanthanides, 3) the assignment of the diamagnetic state is known or readily accessible, 4) selective isotope labeling schemes are available or 5) metal exchange rates occur in a regime that is suitable for exchange experiments. Since the metal exchange rate often can be adjusted by suitably chosen solution conditions (metal ion concentrations in particular) ${ }^{[29,33]}$ the exchange experiments promise to become an important tool for the study of metalloproteins.

\section{Acknowledgements}

We are indebted to Dr. A. Y. Park for the preparation of numerous excellent protein samples. M.J. thanks the Humboldt Foundation for a Feodor-Lynen fellowship. Financial support from the Australian Research Council for a Federation Fellowship for G.O. and the $800 \mathrm{MHz} N \mathrm{MR}$ spectrometer at the ANU is gratefully acknowledged.

Keywords: chemical exchange - metalloproteins paramagnetic relaxation enhancement - pseudocontact shift resonance assignment

[1] I. Bertini, C. Luchinat, G. Parigi, Prog. Nucl. Magn. Reson. Spectrosc. 2002, 40, 249-273.

[2] I. Bertini, A. Donaire, B. Jiménez, C. Luchinat, G. Parigi, M. Piccioli, L. Poggi, J. Biomol. NMR 2001, 21, 85-98.

[3] K. Tu, M. Gochin, J. Am. Chem. Soc. 1999, 121, 9276-9285.

[4] J. Iwahara, D. E. Anderson, E. C. Murphy, G. M. Clore, J. Am. Chem. Soc. 2003, 125, 6634-6635.

[5] I. Díaz-Moreno, A. Díaz-Quintana, M. A. De La Rosa, M. Ubbink, J. Biol. Chem. 2005, 280, 18908-18915.

[6] G. Pintacuda, M. Keniry, A. Y. Park, N. E Dixon, G. Otting, J. Am. Chem. Soc. 2006, 128, 3696-3702.

[7] M. John, G. Pintacuda, A. Y. Park, N. E. Dixon, G. Otting, J. Am. Chem. Soc. 2006, 128, 12910-12916.

[8] A. N. Volkov, J. A. R. Worrall, E. Holtzmann, M. Ubbink, Proc. Natl. Acad. Sci. USA 2006, 103, 18945-18950.

[9] G. Pintacuda, M. John, X.-C. Su, G. Otting, Acc. Chem. Res. 2007, 40, $206-212$.

[10] R. J. Kurland, B. R. McGarvey, J. Magn. Reson. 1970, 2, 268-272.

[11] L. Banci, I. Bertini, G. Cavallaro, A. Giachetti, C. Luchinat, G. Parigi, J. Biomol. NMR 2004, 28, 249-261.

[12] M. John, A. Y. Park, G. Pintacuda, N. E. Dixon, G. Otting, J. Am. Chem. Soc. 2005, 127, 17190-17191.

[13] I. Bertini, C. Del Bianco, I. Gelis, N. Katsaros, C. Luchinat, G. Parigi, M. Peana, A. Provenzani, M. A. Zoroddu, Proc. Natl. Acad. Sci. USA 2004, $101,6841-6846$.

[14] C. Eichmüller, N. R. Skrynnikov, J. Biomol. NMR 2007, 37, 79-95.

[15] H. Senn, K. Wüthrich, Quart. Rev. Biophys. 1985, 18, 111-134. 
[16] G. Pintacuda, M. Keniry, T. Huber, A. Y. Park, N. E. Dixon, G. Otting, J. Am. Chem. Soc. 2004, 126, 2963-2970.

[17] M. Guéron, J. Magn. Reson. 1975, 19, 58-66.

[18] M. Sattler, J. Schleucher, C. Griesinger, Prog. Nucl. Magn. Reson. Spectrosc. 1999, 34, $93-158$

[19] V. Tugarinov, R. Muhandiram, A. Ayed, L. E. Kay, J. Am. Chem. Soc. 2002, $124,10025-10035$.

[20] S. Balayssac, B. Jiminez, M. Piccioli, J. Biomol. NMR 2006, 34, 63-73.

[21] W. Bermel, I. Bertini, I. C. Felli, M. Piccioli, R. Pierattelli, Prog. Nucl. Magn. Reson. Spectrosc. 2006, 48, 25-45.

[22] I. Baig, I. Bertini, C. Del Bianco, Y. K. Gupta, Y.-M. Lee, C. Luchinat, A. Quattrone, Biochemistry 2004, 43, 5562-5573.

[23] C. Schmitz, M. John, A. Y. Park, N. E. Dixon, G. Otting, G. Pintacuda, T. Huber, J. Biomol. NMR 2006, 35, 79-87.

[24] M. John, C. Schmitz, A. Y. Park, N. E. Dixon, T. Huber, G. Otting, J. Am. Chem. Soc., unpublished results.

[25] B. D. Nguyen, Z. Xia, D. C. Yeh, K. Vyas, H. Deaguero, G. N. La Mar, J. Am. Chem. Soc. 1999, 121, 208-217.
[26] I. Bertini, I. C. Felli, C. Luchinat, J. Magn. Reson. 1998, 134, 360-364.

[27] S. Hamdan, P. D. Carr, S. E. Brown, D. L. Ollis, N. E. Dixon, Structure 2002, $10,535-546$

[28] J. Jeener, B. H. Meier, P. Bachmann, R. R. Ernst, J. Chem. Phys. 1979, 71, 4546- 4553.

[29] M. John, M. Headlam, N. E. Dixon, G. Otting, J. Biomol. NMR 2007, 37, 43-51.

[30] J. D. Epperson, L.-J. Ming, Biochemistry 2000, 39, 4037-4045.

[31] N. A. Farrow, O. Zhang, J. D. Forman-Kay, L. E. Kay, J. Biomol. NMR 1994, 4, 727-734.

[32] M. John, A. Y. Park, N. E. Dixon, G. Otting, J. Am. Chem. Soc. 2007, 129, $462-463$.

[33] R. Barbieri, P. J. Hore, C. Luchinat, R. Pierattelli, J. Biomol. NMR 2002, 23, 303-309.

Received: July 27, 2007

Published online on October 1, 2007 